\title{
Nail Unit Squamous Cell Carcinoma: Updates on Diagnosis, Surgical Approach, and the Use of Mohs Micrographic Surgery
}

\author{
Mohammed Dany, MD, PhD
}

\section{RESIDENT PEARLS}

- The diagnosis of nail unit squamous cell carcinoma often is delayed due to its clinical presentation, which frequently mimics benign nail conditions.

- Treatment includes wide local excision, Mohs micrographic surgery, digital amputation, cryotherapy, and topical modalities.
Nail unit squamous cell carcinoma (NSCC) is a malignant neoplasm that can arise from any part of the nail unit including the nail bed, nail matrix, nail groove, and nail fold. Nail unit SCC is the most common malignant nail neoplasm, and yet its diagnosis often is delayed, partly due to the clinical presentation of NSCC mimicking benign conditions such as onychomycosis, warts, and paronychia. Nail unit SCC has a low rate of metastasis; however, a delayed diagnosis often can result in local destruction and bone invasion. It is imperative for dermatologists who are early in their training to recognize this entity and refer for treatment. Many approaches have been used to treat NSCC, including wide local excision, digital amputation, cryotherapy, topical modalities, and recently Mohs micrographic surgery (MMS).

Cutis. 2020;106:E11-E14.

N ail unit squamous cell carcinoma (NSCC) is a malignant neoplasm that can arise from any part of the nail unit. Diagnosis often is delayed due to its clinical presentation mimicking benign conditions such as onychomycosis, warts, and paronychia. Nail unit SCC has a low rate of metastasis; however, a delayed diagnosis often can result in local destruction and bone invasion. It is imperative for dermatologists who are early in their training to recognize this entity and refer for treatment. Many approaches have been used to treat NSCC, including wide local excision, digital amputation, cryotherapy, topical modalities, and recently Mohs micrographic surgery (MMS). This article provides an overview of the clinical presentation and diagnosis of NSCC, the role of human papillomavirus (HPV) in NSCC pathogenesis, and the evidence supporting surgical management.

\section{NSCC Clinical Presentation and Diagnosis}

Nail unit squamous cell carcinoma is a malignant neoplasm that can arise from any part of the nail unit including the nail bed, matrix, groove, and nail fold. ${ }^{1}$ Although NSCC is the most common malignant nail neoplasm, its diagnosis often is delayed partly due to the clinical presentation of NSCC mimicking benign conditions such as onychomycosis, warts, and paronychia., ${ }^{2,3}$ Nail unit SCC most commonly is mistaken for verruca vulgaris, and thus it is important to exclude malignancy in nonresolving verrucae of the fingernails or toenails. Another reason for a delay in the diagnosis is the painless and often asymptomatic presentation of this tumor, which keeps patients from seeking care. ${ }^{4}$ While evaluating a subungual lesion, dermatologists should keep in mind red flags that would prompt a biopsy to

From the Department of Dermatology, University of Pennsylvania, Philadelphia.

The author reports no conflict of interest.

Correspondence: Mohammed Dany, MD, PhD, 3600 Spruce St, 2 Maloney, Philadelphia, PA 19104 (mohammed.dany@pennmedicine.upenn.edu). doi:10.12788/cutis.0122 
rule out NSCC (Table 1), including chronic nonhealing lesions, nail plate nodularity, known history of infection with HPV types 16 and 18, history of radiation or arsenic exposure, and immunosuppression. Table 2 lists the differential diagnosis of a persisting or nonhealing subungual tumor.

Nail unit SCC has a low rate of metastasis; however, a delayed diagnosis often can result in local destruction and bone invasion. ${ }^{5}$ Based on several reports, NSCC more commonly is found in middle-aged and older individuals, has a male predilection, and more often is seen on fingernails than toenails. ${ }^{1,2,6}$ Figure A shows an example of the clinical presentation of NSCC affecting the right thumb.

Although there often is a delay in the presentation and biopsy of NSCC, no correlation has been observed between time to biopsy and rate of disease invasion and recurrence. ${ }^{7}$ Nevertheless, Starace et $\mathrm{al}^{7}$ noted that a low threshold for biopsy of nail unit lesions is necessary. It is recommended to perform a deep shave or a nail matrix biopsy, especially if matrical involvement is suspected. ${ }^{8}$ Patients should be closely followed after a diagnosis of NSCC is made, especially if they are immunocompromised or have genetic skin cancer syndromes, as multiple NSCCs can occur in the same individual. ${ }^{9}$ For instance, one report discussed a patient with xeroderma pigmentosum who developed 3 separate NSCCs. Interestingly, in this patient, the authors suspected HPV as a cause for the field cancerization, as 2 of 3 NSCCs were noted on initial histopathology to have arisen from verrucae. ${ }^{10}$

\section{Histologic Features}

A biopsy from an NSCC tumor shows features similar to cutaneous SCC in the affected areas (ie, nail bed, nail matrix, nail groove, nail fold). Characteristic histologic findings include tongues or whorls of atypical squamous epithelium that invade deeply into the dermis. ${ }^{11}$ The cells appear as atypical keratinocytes, exhibit distinct intracellular bridges, and possess hyperchromatic and pleomorphic nuclei with dyskeratosis and keratin pearls within the dermis. ${ }^{12}$ Immunoperoxidase staining for cytokeratin AE1/AE3 can be helpful to confirm the diagnosis and assess whether the depth of invasion involves the bone. ${ }^{13}$ Figures B and C demonstrate the histopathology of NSCC biopsied from the tumor shown in Figure A.

\section{Role of HPV in NSCC Pathogenesis}

There is no clear pathogenic etiology for NSCC; however, there have been some reports of HPV as a risk factor. Shimizu et $\mathrm{al}^{14}$ reviewed 136 cases of HPVassociated NSCC and found that half of the cases were associated with high-risk HPV. They also found that $24 \%$ of the patients with NSCC had a history of other HPV-associated diseases. As such, the authors hypothesized that there is a possibility for genitodigital HPV transmission and that NSCC could be a reservoir for sexually transmitted high-risk HPV. ${ }^{14}$ Other risk factors are
TABLE 1. Red Flags While Evaluating a Subungual Lesion

Nonhealing or persistent

Irregular nail plate hyperkeratosis or nodularity

History of infection with high-risk HPV

Exposure to radiation

Exposure to arsenic

Immunosuppression

Abbreviation: HPV, human papillomavirus.

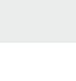

TABLE 2. Differential Diagnosis for a Nonhealing or Persistent Subungual Lesion

\begin{tabular}{ll}
\hline Benign & Malignant \\
\hline Glomus tumor & Bowen disease of the nail unit \\
Periungual fibroma & NSCC \\
Soft tissue chondroma & Subungual amelanotic melanoma \\
Subungual schwannoma & Subungual keratoacanthoma \\
Viral wart & \\
\hline
\end{tabular}

Abbreviation: NSCC, nail unit squamous cell carcinoma.

radiation exposure, chemical insult, and chronic trauma. ${ }^{15}$ The higher propensity for fingernails likely is reflective of the role of UV light exposure and infection with HPV in the development of these tumors. ${ }^{14,15}$

\section{Treatment Options for NSCC}

Several nonsurgical approaches have been suggested to treat NSCC, including topical agents, cryotherapy, $\mathrm{CO}_{2}$ laser, and photodynamic therapy. ${ }^{3,16}$ Unfortunately, there are no large case series to demonstrate the cure rate or effectiveness of these methods..$^{17}$ In one study, the authors did not recommend use of photodynamic therapy or topical modalities such as imiquimod cream $5 \%$ or fluorouracil cream $5 \%$ as first-line treatments of NSCC due to the difficulty in ensuring complete treatment of the sulci of the lateral and proximal nail folds. ${ }^{18}$

More evidence in the literature supports surgical approaches, including wide local excision, MMS, and digital amputation. Clinicians should consider relapse rates and the impact on digital functioning when choosing a surgical approach.

For wide local excisions, the most common approach is en bloc excision of the nail unit including the lateral nail folds, the proximal nail fold, and the distal nail fold. The excision starts with a transverse incision on the base of the 

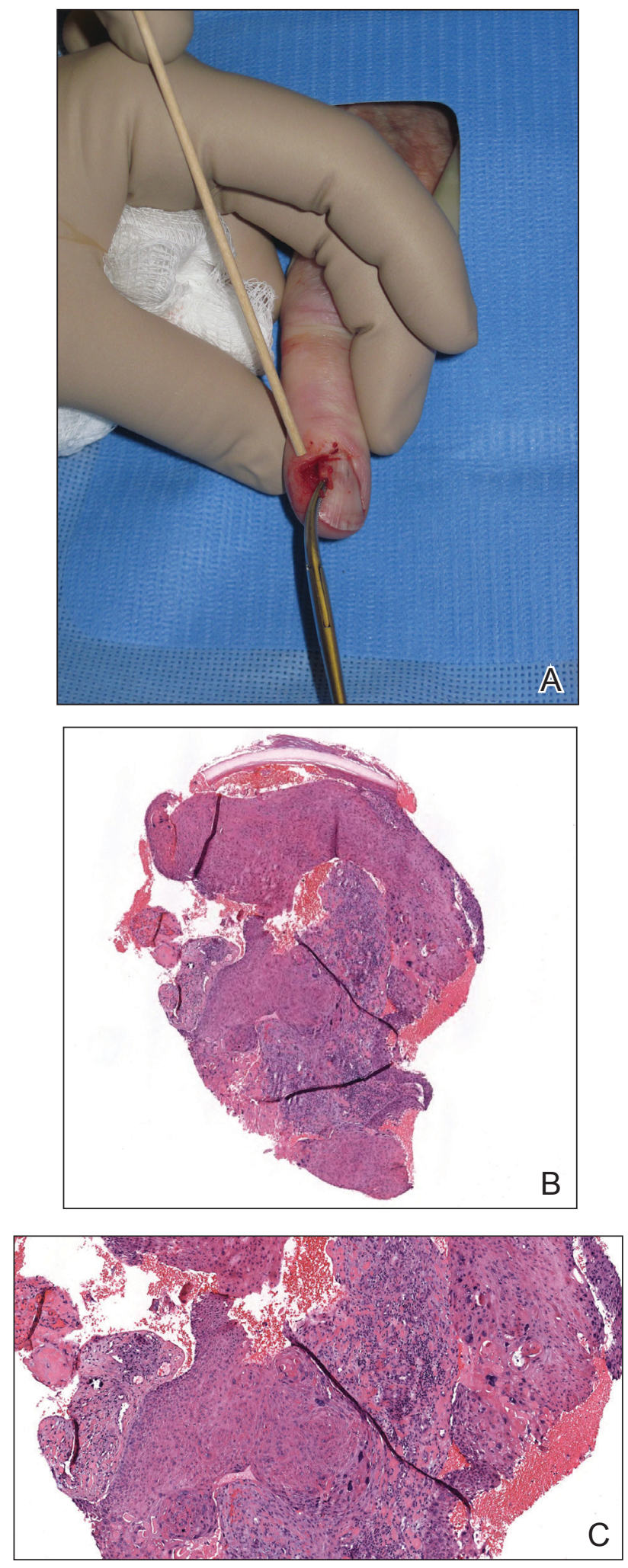

A, Nail unit squamous cell carcinoma (NSCC) tumor prior to performing a biopsy. B and C, Histopathology of NSCC biopsied from the tumor showed atypical keratinocytes in the epidermis extending to the dermis ( $\mathrm{H} \& \mathrm{E}$, original magnifications $\times 30$ and $\times 80$ ). Images courtesy of Adam I. Rubin, MD (Philadelphia, Pennsylvania). distal phalanx, which is then prolonged laterally and distally to the distal nail fold down to the bone. After the incision is made to the depth of the bone, the matrical horns are destroyed by electrocoagulation, and the defect is closed either by a full-thickness skin graft or secondary intent. ${ }^{19}$

Topin-Ruiz et $\mathrm{al}^{19}$ followed patients with biopsyproven NSCC without bone invasion who underwent en bloc excision followed by full-thickness skin graft. In their consecutive series of 55 patients with 5 years of follow-up, the rate of recurrence was only $4 \%$. There was a low rate of complications including graft infection, delayed wound healing, and severe pain in a small percentage of patients. They also reported a high patient satisfaction rate. ${ }^{19}$ Due to the low recurrence rate, this study suggested that total excision of the nail unit followed by a full-thickness skin graft is a safe and efficient treatment of NSCC without bone involvement. Similarly, in another case series, wide local excision of the entire nail apparatus had a relapse rate of only $5 \%$, in contrast to partial excision of the nail unit with a relapse of $56 \% .^{20}$ These studies suggest that wide nail unit excision is an acceptable and effective approach; however, in cases in which invasion cannot be ruled out, histologic clearance would be a reasonable approach. ${ }^{21}$ As such, several case series demonstrated the merits of MMS for NSCC. de Berker et $\mathrm{al}^{22}$ reported 8 patients with NSCC treated using slow MMS and showed tumor clearance after a mean of 3 stages over a mean period of 6.9 days. In all cases, the wounds were allowed to heal by secondary intention, and the distal phalanx was preserved. During a mean follow-up period of 3.1 years, no recurrence was seen, and involved digits remained functional. ${ }^{22}$

Other studies tested the efficacy of MMS for NSCC. Young et $\mathrm{al}^{23}$ reported the outcomes of 14 NSCC cases treated with MMS. In their case series, they found that the mean number of MMS surgical stages required to achieve histologic clearance was 2, while the mean number of tissue sections was $4 .^{23}$ All cases were allowed to heal by secondary intent with excellent outcomes, except for 1 patient who received primary closure of a small defect. They reported a $78 \%$ cure rate with an average time to recurrence of 47 months. ${ }^{23}$ In a series of 42 cases of NSCC treated with MMS, Gou et a ${ }^{17}$ noted a cure rate close to $93 \%$. In their study, recurrences were observed in only 3 patients (7.1\%). These recurrent cases were then successfully treated with another round of MMS. ${ }^{17}$ This study's cure rate was comparable to the cure rate of MMS for SCC in other cutaneous areas. Goldminz and Bennett ${ }^{24}$ demonstrated a cure rate of $92 \%$ in their case series of 25 patients. Two patients developed recurrent disease and were treated again with MMS resulting in no subsequent recurrence. In this study, the authors allowed all defects to heal by secondary intention and found that there were excellent cosmetic and functional outcomes. ${ }^{24}$ Dika et $\mathrm{al}^{25}$ evaluated the long-term effectiveness of MMS in the treatment of NSCC, in particular its ability to reduce the number of digital amputations. Fifteen patients diagnosed with NSCC were treated with MMS as 
the first-line surgical approach and were followed for 2 to 5 years. They found that in utilizing MMS, they were able to avoid amputations in 13 of 15 cases with no recurrence in any of these tumors. Two cases, however, still required amputation of the distal phalanx. ${ }^{25}$

Although these studies suggest that MMS achieves a high cure rate ranging from $78 \%$ to $93 \%$, it is not yet clear in the literature whether MMS is superior to wide local excision. More studies and clinical trials comparing these 2 surgical approaches should be performed to identify which surgical approach would be the gold standard for NSCC and which select cases would benefit from MMS as first-line treatment.

\section{Final Thoughts}

Nail unit SCC is one of the most common nail unit malignancies and can mimic several benign entities. Dermatologists who are early in their training should consider biopsy of subungual lesions with certain red flags (Table 1). It is important to diagnose NSCC for early intervention. Referral for wide local excision or MMS would be ideal. There are data in the literature supporting both surgical approaches as being effective; however, there are no trials comparing both approaches. Distal amputation should be considered as a last resort when wide local excision is not reasonable or when MMS fails to achieve clear margins, thereby reducing unnecessary amputations and patient morbidity. ${ }^{17}$

\section{REFERENCES}

1. Dika E, Starace M, Patrizi A, et al. Squamous cell carcinoma of the nail unit: a clinical histopathologic study and a proposal for classification. Dermatol Surg. 2019;45:365-370.

2. Lee TM, Jo G, Kim M, et al. Squamous cell carcinoma of the nail unit: a retrospective review of 19 cases in Asia and comparative review of Western literature. Int J Dermatol. 2019;58:428-432.

3. Tambe SA, Patil PD, Saple DG, et al. Squamous cell carcinoma of the nail bed: the great mimicker. JCutan Aesthet Surg. 2017;10:59-60.

4. Perrin C. Tumors of the nail unit. a review. part II: acquired localized longitudinal pachyonychia and masked nail tumors. Am J Dermatopathol. 2013;35:693-712.

5. Li PF, Zhu N, Lu H. Squamous cell carcinoma of the nail bed: a case report. World J Clin Cases. 2019;7:3590-3594.

6. Kaul S, Singal A, Grover $C$, et al. Clinical and histological spectrum of nail psoriasis: a cross-sectional study. J Cutan Pathol. 2018;45:824-830.
7. Starace M, Alessandrini A, Dika E, et al. Squamous cell carcinoma of the nail unit. Dermatol Pract Concept. 2018;8:238-244.

8. Kelly KJ, Kalani AD, Storrs S, et al. Subungual squamous cell carcinoma of the toe: working toward a standardized therapeutic approach. J Surg Educ. 2008;65:297-301.

9. Ormerod E, De Berker D. Nail unit squamous cell carcinoma in people with immunosuppression. Br J Dermatol. 2015;173:701-712.

10. Ventéjou S, Bagny K, Waldmeyer J, et al. Skin cancers in patients of skin phototype $\mathrm{V}$ or VI with xeroderma pigmentosum type $\mathrm{C}(\mathrm{XP}-\mathrm{C})$ : a retrospective study. Ann Dermatol Venereol. 2019;146:192-203.

11. Mikhail GR. Subungual epidermoid carcinoma. J Am Acad Dermatol. 1984;11:291-298

12. Lecerf $\mathrm{P}$, Richert $\mathrm{B}$, Theunis A, et al. A retrospective study of squamous cell carcinoma of the nail unit diagnosed in a Belgian general hospital over a 15-year period. J Am Acad Dermatol. 2013;69:253-261.

13. Kurokawa I, SenbaY, Kakeda M, et al. Cytokeratin expression in subungual squamous cell carcinoma. J Int Med Res. 2006;34:441-443.

14. Shimizu A, Kuriyama $Y$, Hasegawa $M$, et al. Nail squamous cell carcinoma: a hidden high-risk human papillomavirus reservoir for sexually transmitted infections. J Am Acad Dermatol. 2019;81:1358-1370.

15. Tang N, Maloney ME, Clark AH, et al. A retrospective study of nail squamous cell carcinoma at 2 institutions. Dermatol Surg. 2016;42(suppl 1):S8-S17.

16. An Q, Zheng S, Zhang L, et al. Subungual squamous cell carcinoma treated by topical photodynamic therapy. Chin Med J (Engl). 2020;133:881-882.

17. Gou D, Nijhawan RI, Srivastava D. Mohs micrographic surgery as the standard of care for nail unit squamous cell carcinoma. Dermatol Surg. 2020;46:725-732.

18. Dika E, Fanti PA, Patrizi A, et al. Mohs surgery for squamous cell carcinoma of the nail unit: 10 years of experience. Dermatol Surg. 2015;41:1015-1019.

19. Topin-Ruiz S, Surinach C, Dalle S, et al. Surgical treatment of subungual squamous cell carcinoma by wide excision of the nail unit and skin graft reconstruction: an evaluation of treatment efficiency and outcomes. JAMA Dermatol. 2017;153:442-448.

20. Dalle S, Depape L, Phan A, et al. Squamous cell carcinoma of the nail apparatus: clinicopathological study of 35 cases. $\mathrm{Br} J$ Dermatol. 2007; 156:871-874.

21. Zaiac MN, Weiss E. Mohs micrographic surgery of the nail unit and squamous cell carcinoma. Dermatol Surg. 2001;27:246-251.

22. de Berker DA, Dahl MG, Malcolm AJ, et al. Micrographic surgery for subungual squamous cell carcinoma. Br J Plast Surg. 1996;49:414-419.

23. Young LC, Tuxen AJ, Goodman G. Mohs' micrographic surgery as treatment for squamous dysplasia of the nail unit. Australas J Dermatol. 2012;53:123-127.

24. Goldminz D, Bennett RG. Mohs micrographic surgery of the nail unit. J Dermatol Surg Oncol. 1992;18:721-726.

25. Dika E, Piraccini BM, Balestri R, et al. Mohs surgery for squamous cell carcinoma of the nail: report of 15 cases. our experience and a longterm follow-up. Br J Dermatol. 2012;167:1310-1314. 